\title{
LA AFECTIVIDAD COMO FUNDAMENTO FENOMENOLÓGICO DE LA COMUNIDAD Y LA EXPERIENCIA DEL OTRO
}

\author{
MYRIAM DÍAZ ERBETTA \\ Pontificia Universidad Católica de Valparaíso
}

\begin{abstract}
RESUMEN: La comprensión y el análisis del problema de la intersubjetividad y la experiencia del otro ha sido un ámbito controvertido para la fenomenología en general, y no es excepción en la fenomenología de la vida de Michel Henry. La fenomenología henryana presenta como núcleo de su reflexión la subjetividad, que es concebida como realidad encarnada (es un cuerpo, una carne), una realidad afectiva y fundamentalmente viviente, cuya praxis se expresa en el trabajo subjetivo viviente. Desde este punto de partida filosófico, la alteridad, la intersubjetividad y la comunidad sólo son posibles para Henry desde la afectividad, que es concebida como el único horizonte de la manifestación, ya no de la subjetividad solamente, sino de toda manifestación posible, es decir, como esencia de la manifestación.
\end{abstract}

PALABRAS CLAVE: Henry; subjetividad; afectividad; otro; comunidad.

\section{Affectivity as phenomenological foundation of community and experience of the other}

ABSTRACT: A controversial area for phenomenology in general and for Michel Henry's philosophy of life in particular, has been the understanding and the analyzing of intersubjectivity and the experience of the other. Henry's phenomenology has as the central engagement of its reflection the subjectivity, conceived as an embodied reality (it is a body, a flesh), an affective and fundamentally living reality, whose praxis is expressed in subjective living work. For Henry's philosophical point of view, otherness, intersubjectivity, and community are possible only from affectivity, conceived as the only horizon of manifestation, not just for subjectivity but for all possible manifestation, that is to say, as the essence of manifestation.

KEY WORDS: Henry; subjectivity; affectivity; other; community.

\section{INTRODUCCIÓN}

En primer lugar resulta fundamental señalar que la filosofía de Michel Henry constituye y puede interpretarse como una filosofía de la subjetividad. A partir del método onto-fenomenológico que ella misma se da, y de su propio devenir, esta filosofía adopta una mirada crítica de la tradición filosófica, y busca instaurar una filosofía de la subjetividad en tanto filosofía primera, de modo de remontar al fundamento donde todo depende sólo de sí mismo y de nada más ${ }^{1}$.

El punto de partida de su análisis es el cuerpo, pues la subjetividad para Henry es concebida como una realidad encarnada, el yo es un cuerpo ${ }^{2}$, y un

1 Cf. Henry, Michel, L'essence de la manifestation, PUF, París, 1963, p. 816.

2 Cf. Henrr, Michel, Philosophie et phénoménologie du corps, PUF, París, 1965, p. 271. 
cuerpo viviente (una carne), en tanto se identifica con la afectividad. La afectividad, a su vez, refiere el poder de sentir, es decir de sentir-se, es un pathos fundamental que nos une a la vida y a la comunidad de vivientes ${ }^{3}$. Toda actividad vital es afectiva, toda acción y comprensión del mundo es afectiva, todo contacto con el otro supone la afectividad, pues es imposible concebir un yo carente de afectividad y de sentimientos.

Si bien en la filosofía henryana el problema de la experiencia del otro y la intersubjetividad pudiese parecer insalvable, dado el concepto de auto-afección y las restricciones que impone el concepto de carne y la imposibilidad de «tocar» la carne en el otro como experiencia subjetiva del propio pathos, Henry propone que la experiencia del otro es posible y encuentra su inteligibilidad sólo en la afectividad, es pathos-con, simpatía ${ }^{4}$. Así vista, la comunidad de vivientes se mantiene unida por la Vida y será identificada con la analogía de una napa subterránea, imagen «mística» que, desde una perspectiva crítica, nos permite advertir el curso posterior del pensamiento henryano dedicado a la filosofía del cristianismo.

Aun con estas dificultades, tanto para la filosofía como para las ciencias humanas y sociales - que han buscado en la fenomenología un fundamento para comprender la vida en común y la experiencia intersubjetiva - la filosofía de Michel Henry es un punto de partida interesante y provocador para pensar la experiencia del otro y la comunidad.

\section{LA SUBJETIVIDAD EN LA FENOMENOLOGÍA DE LA VIDA}

Michel Henry se presenta como un filósofo crítico de lo que ha denominado «monismo ontológico», es decir, aquella idea que ha sido el motor de la historia de la filosofía, y que propone que la única fenomenalidad auténtica se da en el conocimiento objetivo y exterior del mundo. En su crítica a este monismo ontológico, Henry libra un combate en favor de los derechos ontológicos e imprescriptibles de la subjetividad. Ya en un escrito temprano en su «libreta de anotaciones de guerra», con apenas 20 años, Henry escribe:

«19.11.1942- la estructura del mundo es la subjetividad. Pero es preciso dar cuenta, a su vez, de la estructura de la subjetividad. Kant la ha descrito parcialmente; Heidegger lo hizo de modo más completo. Hay que explicarla en su totalidad. $\nu^{5}$

\footnotetext{
3 Cf. Henry, Michel, Incarnation, Seuil, París, 2000, p. 348.

4 Cf. Henry, Michel, Phénoménologie matérielle, PUF, París, 1990, p. 140.

5 Henry, Michel, Libreta de anotaciones de guerra, inédito, citado por LecLerco, Jean, en "Afectividad, pasividad y experiencia pura. Reflexiones sobre el proyecto de Michel Henry», en Lipsitz, Mario y Belvedere, Carlos, Problemas de fenomenología material, UNGS, Buenos Aires, 2016, p. 13.
} 
En síntesis, según sus propias palabras, «la subjetividad es el sentido del mundo» ${ }^{6}$, o dicho de otro modo, la subjetividad es la condición de posibilidad de todo ser y de todo conocimiento, pues la esencia de la manifestación, que Henry identifica con la afectividad, no es más que la vida misma que se expresa en el cuerpo, en el sentimiento y en la praxis.

Para Henry el ser humano no es una cosa más entre las cosas del mundo, sino que es aquel donde se lleva a cabo la manifestación de todas las cosas. La vida se experimenta en una suerte de abrazo patético donde aún no hay objeto, ni mundo, ni «conocimiento»:

«En esta experiencia muda y previa a las cosas es donde le advienen sus propiedades fundamentales, la fuerza, la potencia, la corporeidad, la acción, también un saber mucho más profundo y decisivo que el de la conciencia o el de la ciencia.» ${ }^{7}$

La primera categoría con la que Henry identifica la subjetividad es el cuerpo, en tanto cuerpo viviente. El movimiento de la vida es, por principio, individual, es la propia transformación del individuo. De esta manera, un yo no se diferencia de otro yo por cualidades naturales, psíquicas o espirituales, ni tampoco por el lugar o el tiempo en que nació, pues el principium individuationis no se debe a categorías de la exterioridad. Un yo se diferencia de otro porque es originariamente el mismo y lo es en su auto-afección ${ }^{8}$. No existe, así, una vida anónima, una vida de nadie, es siempre la vida de un yo, de un sí mismo.

Para Henry, la filosofía del sujeto tiene una relevancia fundamental para el conocimiento y también para la existencia misma: «La subjetividad no sólo produce, pues, las idealizaciones del mundo matemático de la ciencia, sino que ante todo, produce el mundo de la vida $»^{9}$. La relevancia de la subjetividad emerge y se evidencia cuando reconocemos que los objetos o entes del mundo son lo que son, gracias al conjunto de operaciones subjetivas que los hacen aparecer y los traen así a la condición de fenómenos:

«La subjetividad es aquello que me da el mundo a cada instante y, si entendemos por mundo todo lo que es, ella es el fundamento de todas las cosas, el absoluto al cual ellas re-envían y sin el cual no serían. ${ }^{10}$

6 Henry, Michel, Libreta de anotaciones de guerra, inédito, citado por LecLerco, Jean y Henry, Anne, «Biographie en forme d'entretien», en Cahier Michel Henry, Lâge de l'homme Paris-Lausanne, 2009, p. 10.

7 Henry, Michel, Introducción a Fenomenología de la Vida, Prometeo, Buenos Aires, 2010, p. 15.

8 Cf. Henry, Michel, Phénoménologie de la vie, Tome I De la phénoménologie, PUF, París, 2003, p. 51.

9 Henry, Michel, Phénoménologie de la vie, Tome IV Sur l'éthique et la religion, PUF, París, 2004, p. 12.

10 Henry, Michel, Phénoménologie de la vie, Tome II De la subjectivité, PUF, París, 2003, p. 25 . 
Entonces, podemos afirmar con Henry que la subjetividad es el fundamento de la fenomenalidad, pues sin ella no habría «mundo» que pudiese aparecer.

\section{CUERPO Y SUBJETIVIDAD}

Dado que el primer concepto que propone Henry para pensar la subjetividad es el «cuerpo», la subjetividad es concebida como una "realidad encarnada», el yo «no tiene un cuerpo, sino que es un cuerpo» ${ }^{11}$. Según Henry, una nueva concepción del sujeto y del cuerpo exigiría una nueva reflexión filosófica de todos los actos «materiales» del hombre, es decir, de todos aquellos actos que implican una actividad directa con el medio trascendente: el trabajo, el rito, el movimiento y posteriormente una revisión de todo el saber y el pensamiento humanos, es decir, de la cultura en sentido amplio ${ }^{12}$. El cuerpo, en su naturaleza originaria, pertenece entonces a una esfera de la existencia que es la subjetividad misma.

En la historia de la filosofía occidental ha primado la separación almacuerpo, con la evidente primacía del alma y la consecuente desvalorización del cuerpo. La temprana obra de Henry Philosophie et phénoménologie du corps (1965) se enmarca en el intento de establecer una fenomenología que aclare el estatuto mismo de la fenomenalidad y del aparecer, y en esta búsqueda, aparece con fuerza el carácter subjetivo del cuerpo. Así como la subjetividad no es mera conciencia, intencionalidad, relación a un objeto, ek-stasis, el cuerpo no es mera acción, sino fundamentalmente pathos. Se reconoce así una pasividad radical como constitutiva del cuerpo.

El texto Incaration: une philosophie de la chair (2000), radicaliza y profundiza esta intuición filosófica temprana. Encarnación designa, para Henry, la condición no sólo de poseer un cuerpo, sino de ser una carne. Ambos conceptos, cuerpo y carne, no son lo mismo, pues mientras cuerpo mienta el cuerpo exterior, carne mienta el cuerpo subjetivo (vivo, capaz de afectar-se). En su filosofía, Henry ha definido la carne como cuerpo viviente, como la expresión de todos los poderes subjetivos y de la afectividad. El cuerpo, así entendido, es el cuerpo exterior, en el sentido de los objetos del mundo, el cuerpo que aparece en el horizonte de la exterioridad, en el aparecer del mundo. La vida, identificada con el cuerpo subjetivo-viviente, por el contrario, se niega a aparecer en el mundo y sólo puede ser vivida desde ella misma, como auto-revelación de sí misma ${ }^{13}$. La carne (cuerpo viviente) se revela como pathos, como ese sufrir y soportarse a sí mismo. Es la vida en su auto-revelación inmanente que es idéntica a todas sus impresiones, haciendo de ellas una sola y misma carne.

11 Henry, Michel, Philosophie et phénoménologie du corps, op.cit., p. 282.

12 Cf., Ibid., p. 303, nota.

13 Cf. Henry, Michel, Phénoménologie de la vie, Tome I De la phénoménologie, op.cit, p. 167. 
La clave de la argumentación de Henry sobre la relevancia fundamental de la carne en la constitución de la subjetividad, entendida como cuerpo viviente, como pathos, estaría en que todo cuerpo sensible (sentido, visto, escuchado, tocado, movido) presupone otro cuerpo que lo siente, que lo ve, que lo toca o lo mueve. Esto implicaría que las operaciones de este último constituyen y hacen posible el primero, convirtiéndolo así en un cuerpo trascendental y constituyente, un cuerpo-sujeto o cuerpo subjetivo, sin el cual el cuerpo-objeto del mundo no podría ser ni aparecer. De este modo, nuestro cuerpo mundanoobjeto es posible sólo en tanto está fundado en la vida o en la carne:

«Solamente nuestra carne nos da acceso a este cuerpo y por tanto al mundo, porque ella es la que nos da primero acceso a sí misma, porque se da impresionalmente a sí allí donde toda auto-donación se realiza, en la vida y por ella.» ${ }^{14}$

Como hemos afirmado, esta concepción del cuerpo tendría, a su vez, consecuencias no sólo ontológicas, sino también éticas y epistemológicas, si concebimos el cuerpo como un saber, tal como propone Henry. La argumentación que sustenta esta constitución trascendental del cuerpo viviente se funda en que la vida no necesita pasos previos para revelarse, sino que puede dar cuenta de la acción de un modo inmediato. Se trata de una experiencia inmanente y trascendental, que identifica ego y cuerpo.

La esencia de la vida sensible y sus vivencias intencionales, no es otra cosa que la vida de la subjetividad y del cuerpo subjetivo en su auto-afección. En este sentido, el mundo mismo, no sólo nuestra propia existencia, es siempre tonalizado por las afecciones internas de nuestro propio cuerpo, un cuerpo subjetivo y viviente en sentido fenomenológico. Se trata entonces, de un «cosmos viviente» diferente a la idea husserliana y heideggeriana de mundo, de un espacio de alegría y sufrimiento que somos nosotros mismos, antes de ser objeto de conocimiento ${ }^{15}$.

Las principales críticas a la filosofía henryana del cuerpo apuntan al solipsismo y a la comprensión del cuerpo subjetivo inmanente como una mónada cerrada sobre sí misma, así como el haberse centrado en una experiencia que descarta la trascendencia y el mundo ${ }^{16}$. Estas críticas pueden matizarse o debatirse si recordamos que el ser inmanente de la subjetividad, lejos de formar una mónada en el desierto del mundo, lo habita y lo conoce desde un conocimiento que no es "objetivo» sino desde una inmanencia y una inmediatez que le es dada en cada presencia. El sujeto inmanente, y sólo él, funda la realidad de los objetos que construyen las ciencias físico-químicas en el horizonte del universo natural. Si no fuese así no habría mundo de la vida, sería un mundo anónimo

$14 \quad$ Ibid., p. 171.

15 Cf. Dufour-Kowalska, Gabrielle, Michel Henry: passion et magnificence de la vie, Beauchesne, París, 2003, p. 37.

16 Cf. Moser, Vincent, «Avec Michel Henry: pour une monadologie radicale» en Les cahiers philosophiques de Strasbourg, n³0, 2011, pp. 143-159. 
y desconocido para el viviente ${ }^{17}$. Persisten, no obstante, en la concepción del cuerpo como sujeto, problemas y tensiones que nos parecen complejas, en especial el problema de la experiencia del otro, que veremos a continuación.

\section{El CUERPo y LA EXPERIENCIA DEL OtRo}

El problema del acceso a la experiencia del otro y a su cuerpo en una fenomenología de la inmanencia, como la fenomenología de Henry, es un tema complejo. Este problema ya ha sido considerado como tal en la fenomenología en general, incluso por Husserl en Ideas II y en Meditaciones Cartesianas, pero se agudiza cuando la experiencia del cuerpo es definida desde la inmanencia de una corporeidad originaria subjetiva.

Henry aborda tempranamente el problema del otro en su texto Philosophie et Phénoménologie du corps, y luego con mayor profundidad en Incarnation. Respecto al cuerpo, el principal problema consiste en cómo transferir la experiencia de un cuerpo vivo, la experiencia de mi cuerpo, que es inmediata e inmanente, al cuerpo de otro, que se me presenta en el ámbito de la sensibilidad, es decir, como exterior, como trascendente, como cuerpo representado. Recordemos que Henry ha definido la experiencia del cuerpo como experiencia interna trascendental, es decir, inmediata e inmanente. La experiencia en la que el otro experimenta lo que le es propio, su propio cuerpo en la inmanencia, escapa a mi percepción directa inmanente y pertenece a su subjetividad, que para mí es inalcanzable. Se trata de una subjetividad absoluta que escapa a toda intencionalidad. Este problema, que es propio de la fenomenología en general, aparece en Husserl asociado a la constitución del alter ego, pero no porque sea un alter, sino fundamentalmente porque es un ego, identificado en Meditaciones cartesianas, como una mónada ${ }^{18}$.

Para Henry la vida trascendental en el viviente no permite ningún distanciamiento, por tanto, toda intencionalidad será incapaz de dar acceso a esa otra vida, a esa otra carne ${ }^{19}$. El problema que ve Henry en la filosofía de Husserl estaría en que su filosofía presenta un enfoque «perceptivo» e intencional que también se imprime a la experiencia del otro convirtiéndolo en «cosa», en cuerpo objeto ${ }^{20}$. Husserl de ese modo quedaría atrapado en las leyes de la percepción y no del pathos.

Este problema de la experiencia del otro tiene consecuencias, como veremos más adelante, a la hora de definir la comunidad. Henry estudia este problema

17 Cf. Dufour-Kowalska, Gabrielle, op.cit., p. 40.

18 Cf. Husserl, Edmund, Meditaciones cartesianas, Fondo de Cultura Económica, Madrid, 1985, p. 154.

19 Cf. Fainstein, Graciela, Michel Henry: una fenomenología de la afectividad o una filosofía en el límite del pensamiento, Tesis para la obtención del grado de Doctor, Pontificia Universidad Comillas de Madrid, Madrid, 2009, p. 223.

20 Henry, Michel, Phénoménologie matérielle, op.cit., p. 152. 
partiendo del contexto del amor y del erotismo. En la relación erótica de los amantes, aparece el deseo que se convierte en pulsión, en la fuerza del afecto, de modo que el deseo quiere llegar a tocar la sensación del otro en su cuerpo y en su vida, en su carne. Henry reconocerá finalmente la imposibilidad de llegar a ese cuerpo subjetivo del otro, donde habita su vida propia. Precisamente será esta imposibilidad la que marca la angustia y el fracaso de la unión carnal en lo que Henry denomina «la noche de los amantes» ${ }^{21}$. El amor erótico fracasa en el mundo producto de la imposibilidad de tener una experiencia de inmanencia absoluta de la vida encarnada del otro. No podemos experimentar el cuerpo del otro tal como él lo experimenta en su vida inmanente, no tenemos acceso a esa experiencia de placer o dolor tal como él la experimenta, pues: «El cuerpo subjetivo original está constantemente presente a sí mismo, en esta Archi-presencia de la Vida ${ }^{22}$. La inmersión de cada uno en su propio cuerpo sostiene la inmersión de la vida en ella misma, vida que para el viviente, para ese sí individual y concreto, es una experiencia patética que ninguna fuerza puede romper.

La problemática que plantea el acceso a la experiencia corporal del otro, no tiene sólo un carácter negativo, sino que pone de relieve la dimensión ética del cuerpo del otro, que en tanto tal, no puede ser nunca tratado como objeto, aunque sea éste el único acceso que tenemos a él en el mundo. Tenemos acceso al cuerpo objetivo del otro, pero a lo que no tenemos acceso es a su experiencia corporal subjetiva, que es la experiencia de la carne habitada por la vida, una vida que hace de él un sí mismo particular. Esta limitación puede tener implicancias éticas necesarias de reflexionar en ámbitos como la medicina, las construcciones de ideales de belleza, los derechos reproductivos, el género, etc.

Volveremos sobre este punto a propósito de la comprensión de la afectividad y la intersubjetividad, dada su relevancia para la filosofía y las ciencias sociales.

\section{LA SUBJETIVIDAD COMO AFECTIVIDAD}

La ontología de la vida subjetiva que desarrolla Henry define la afectividad e inmanencia como la esencia de la vida misma. La totalidad de nuestra experiencia, se compone de una vida expresada a través innumerables tonalidades del sufrimiento y el gozo, que se renuevan a cada instante en el pathos esencial de la subjetividad humana. Por esta razón para Henry hay identidad entre los conceptos de subjetividad, cuerpo subjetivo, afectividad y vida.

Henry distingue entre sensibilidad y afectividad: la sensibilidad corresponde al poder de sentir «algo», alguna cosa, y ser afectados por ella, es decir, nos remite al concepto de intencionalidad. La afectividad en cambio es la forma en la cual ésta es afectada no por otra cosa, sino por ella misma. Esta afección original es una autoafección, es el sentimiento de sí que la constituye (ipseidad),

21 Henry, Michel, Incarnation, op.cit., p. 300.

22 Henry, Michel, Phénoménologie de la vie, Tome I De la phénoménologie, op.cit., p. 163. 
es fundamentalmente la capacidad de sentir-se. La posibilidad de ser afectado por cualquier cosa del mundo -incluso por un otro- reside precisamente en esa afectividad originaria que hace la ipseidad de sí, pues la afectividad funda la sensibilidad: «la afectividad es la condición de la sensación constituyendo su realidad misma y la sustancia de su ser fenomenológico efectivo y concreto ${ }^{23}$.

El aporte de Henry consiste justamente en haber puesto de relieve el carácter afectivo del sujeto y haber reconocido que toda relación con el mundo es una relación originariamente afectiva. Todo acto representacional implica así la afectividad de aquel que lo vive. Todas las modalidades de la vida representativa son, finalmente, modalidades de la vida afectiva y deben ser tomadas como tal ${ }^{24}$.

La afectividad es una experiencia de inmanencia e interioridad radical, pues lo que se revela en el sentimiento es el sentimiento mismo, no un contenido trascendente. Así mismo, los sentimientos y la afectividad son posibles sólo porque existe un viviente que los vive, los siente, los experimenta y es capaz de ser afectado por ellos, teniendo en cuenta que lo que lo afecta y lo afectado son una identidad ${ }^{25}$. La afectividad es para Henry nuestra esencia, nuestra pasividad original. La pasividad se expresa en este «dejà», este "ya dado» del sentimiento que manifiesta su impotencia, su imposibilidad de librarse de sí mismo que lo sumerge en su efectividad ${ }^{26}$. Los sentimientos, la cólera, el amor, el aburrimiento, etc., como experiencias subjetivas, no pueden ser comprendidos más que como una modalidad radical de la vida de un «sí».

Si identificamos subjetividad, afectividad e ipseidad, es posible reconocer que somos nuestros propios sentimientos. No se trata de que «tengamos» sentimientos como si estos fuesen un contenido de conciencia, otra cosa diferente de nosotros mismos, sino que se trata de modalidades de la vida subjetiva misma, pues la afectividad se constituye en los actos subjetivos mismos: no hay sentiente y sentido (sujeto y contenido intencional). Todos los actos subjetivos son afectivos, incluso los actos racionales y de juicio, y todo juicio, en tanto es habitado por la vida de un sí, es por consecuencia radicalmente afectivo ${ }^{27}$. Incluso la propia actividad científica tendría, como afirma Henry en La Barbarie, una realidad fenomenológica en la afectividad originaria de la vida. Hablar de una vida científica es posible sólo porque existe una vida trascendental sin la cual ella no existiría, porque es una expresión o una modalidad de la vida originaria ${ }^{28}$.

Toda experiencia del sujeto es esencialmente afectiva y está fundada en una tonalidad. El mundo -incluidos los otros- se nos aparece como afectivo porque adviene en sus tonalidades sobre el fondo de su esencia siempre afectiva. Las cosas se nos presentan como agradables, desagradables, amables, deseables, etc.,

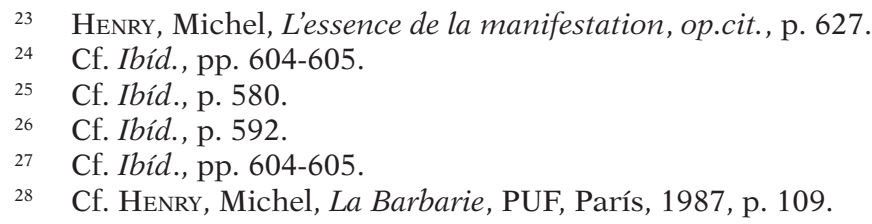


es decir, provocan agrado, deseo, aversión, etc. Si las cosas provocan en nosotros, como sujetos, estos sentimientos, no es por ellas mismas, sino que es a causa del sentimiento que constituye esa presencia, el sentimiento que se siente a sí mismo y nos abre el mundo desde la afectividad. Henry lo expresa del siguiente modo:

«Todo comportamiento, toda acción, toda percepción, todo conocimiento, toda modalidad que implica un objeto y su relación, todas las modalidades de la vida representativa son indisolublemente modalidades de la vida afectiva y deben ser tomadas como tales. $\aleph^{29}$

Esta perspectiva, sin embargo, conlleva una paradoja pues, si la vida afectiva es una experiencia inmanente, intentar someter los sentimientos al poder del pensamiento significa proyectarlos en el medio del ser trascendente, introducirlos en la estructura de la conciencia como correlato, como objeto, lo que es imposible en la filosofía henryana. El carácter invisible del sentimiento y la afectividad es abordado por Henry en varias de sus obras, por ejemplo, en su libro sobre Kandinsky, y fundamentalmente en L'essence de la manifestation. La revelación de la afectividad es una revelación escondida, extraña a la luz del mundo, porque se trata del experimentarse a sí mismo del sentimiento, en la interioridad de la vida misma.

En Voir l'invisible. Sur Kandinsky, Henry retoma la distinción entre los dos modos fundamentales de la manifestación, el exterior o del mundo y el interior o inmanencia, y plantea que lo interior se revela a la manera de la vida:

«La vida se siente y se experimenta ella misma de forma inmediata, de modo que coincide consigo misma en cada punto de su ser y, sumergida toda entera en sí y agotándose en ese sentimiento de sí, se realiza como un pathos. La manera en que lo Interior se revela a sí mismo, en que la vida se vive a sí misma, en que la impresión se impresiona inmediatamente a sí misma, en que el sentimiento se afecta a sí mismo —antes de cualquier mirada e independientemente de ella- es la afectividad. ${ }^{30}$

En este sentido, Henry plantea que el esfuerzo que hace Kandinsky con su pintura abstracta es expresar ese interior, la vida, lo invisible y lo describe en la siguiente ecuación:

$$
\text { «Interior }=\text { interioridad }=\text { invisible }=\text { vida }=\text { pathos }=\text { abstracto. } .{ }^{31}
$$

Se trata entonces de la constante venida a sí misma de la vida, su surgimiento interior continuo. Abstracto para Henry (en referencia al arte) no quiere decir que procede del mundo en un proceso de simplificación, sino que refiere eso que estaba antes que el mundo y que no tiene necesidad de él para ser: «la vida que se abraza en la noche de su subjetividad radical, donde no hay luz ni mundo $»^{32}$.

29 Henry, Michel, L'essence de la manifestation, op.cit., pp. 603-604.

30 Henry, Michel, Voir l'invisible. Sur Kandinsky, PUF, París, 2005, p. 18.

31 Ibíd.

32 Ibid., p. 33. 
Sólo la vida se relaciona consigo misma en auto-afección, pues sólo hay acceso a la vida desde el interior de ella y por medio de ella: «Decíamos, ningún camino lleva a la vida sino ella misma. Es en la vida donde hay que mantenerse para tener acceso a ella, es desde ella desde donde hay que partir» ${ }^{33}$.

Según Henry el pensamiento no dispone de la dimensión ontológica a la que pertenece el sentimiento, pues antes de que la mirada reflexiva del pensamiento tenga lugar, el sentimiento ya está ahí, a la sombra, en la oscuridad. Esto plantea, sin embargo, una dificultad y una posible aporía a la hora de intentar una filosofía de la afectividad. La imposibilidad del pensamiento para comprender la afectividad implicaría abordarla desde una dimensión distinta del pensar racional, lo que nos llevaría a una suerte de filosofía en el límite de lo posible $^{34}$. Esta imposibilidad empuja a Henry a pensar en la experiencia del arte, la danza, la literatura ${ }^{35}$, o en la pintura abstracta de Kandisnky, que no busca la representación. Sólo este lenguaje de la vida permite «narrar» el propio pathos y permite fundar el lenguaje objetivo del mundo trascendente ${ }^{36}$.

Finalmente el propio Henry, buscando esta aproximación a la vida fuera de la representación y la razón, se ha aproximado al arte y a la religión, y reconoce que quizás la única salida a este problema esté en la estética, la religión y la ética, que le parecen las formas más perfectas y elevadas de la cultura, las que muestran la vida como tal ${ }^{37}$.

\section{LA AFECTIVIDAD Y EL PROBLEMA DEL OTRO}

Hemos abordado el problema del otro respecto del cuerpo, a propósito de la imposibilidad de acceder a la experiencia inmanente del cuerpo subjetivo del otro, al problema del «tocar» y la barrera impuesta por el cuerpo objetivo frente a la carne invisible. En el caso de la afectividad, esta imposibilidad problemática adquiere un nuevo sentido: ¿Cómo vivir una afectividad que es fundamentalmente auto-afección? ¿Cómo definir los sentimientos hacia otro si se trata de un sentimiento que se siente a sí mismo? ¿Cómo hacer experiencia del otro en la afectividad? Partiendo desde la inmanencia, Henry parece correr desde el principio el riesgo del solipsismo, pues si la vida es auto-afección, ¿queda espacio para el otro? ${ }^{38}$

Si bien Henry expresó su intención de haber escrito un libro enteramente dedicado a la intersubjetividad, que consideraba un problema de una dificultad

\footnotetext{
33 Ibid., p. 37.

34 Cf. Fainstein, Graciela, op.cit., p. 392.

35 Cf. Henry, Michel, Phénoménologie de la vie, Tome III De l'art et du politique, PUF, París, 2004, p. 314.

36 Cf. Ibíd., p. 340.

37 Cf. Henry, Michel, Phénoménologie de la vie, Tome IV Sur l'éthique et la religion, op.cit., pp. 21-22.

38 Cf. Fainstein, Graciela, op.cit., p. 216.
} 
extraordinaria ${ }^{39}$, abordó el problema del otro en su libro Phénoménologie Materielle. En el apartado titulado «Pathos-con» reflexiona sobre el problema de la intersubjetividad, a propósito de las Meditaciones Cartesianas de Husserl.

El principal problema que observa Henry en la fenomenología husserliana, es que la experiencia del otro se basa en el encuentro del otro en mí, es decir, en mi experiencia a partir de la intencionalidad. Al reconocer al otro como alter-ego en una intencionalidad, éste se transforma en nóema, por tanto para Husserl se presentaría como parte de la teoría de la constitución trascendental del mundo objetivo ${ }^{40}$. Es decir, bajo esta mirada, existe homogeneidad entre la experiencia que tengo del mundo y la que tengo del otro, pues ambas se dan en el contexto de la intencionalidad noético-noemática. Por el contrario, para Henry, la experiencia de la alteridad, tal como se experimenta en sí, se juega en la afectividad, en la emoción ante la reciprocidad del deseo, en el sentimiento de presencia o ausencia, en la soledad, el amor, el odio, el resentimiento, el aburrimiento, el perdón, la exaltación, la tristeza, la alegría, la admiración: «tales son las modalidades concretas de nuestra vida en calidad de vida con el otro, en cuento pathos-con, a título de sim-patía bajo todas sus formas» ${ }^{41}$.

Henry critica a Husserl el no haber dicho nada sobre la afectividad en su "Quinta meditación», quedando ignorada completamente en la constitución de la experiencia del otro, que es considerada así, sólo como experiencia de la intencionalidad. Para Henry en cambio, la afectividad y el sentimiento son las modalidades que posibilitan la experiencia del otro, y no pueden quedar excluidas de ésta. Una condición no-afectiva, como la intencionalidad, no puede ser la condición de algo que resulta necesariamente afectivo, como ocurre con la experiencia del otro. Husserl consideraría la experiencia del otro como parte de la experiencia de los elementos mundanos que constituyen la esfera de «lo mío propio ${ }^{42}$ del ego, es decir, entidades constituidas, un objeto sobre el cual se dirige la mirada. A partir de la constitución del ego, y de lo «mío propio» en calidad de unidad psico-física, el otro entra en mi experiencia como cuerpo que aparece en la esfera de «lo propio», y lo percibo a su vez como organismo, como un cuerpo habitado por un ego constituido. Esto es posible, precisamente, por una analogía que considera como organismo y como cuerpo, el cuerpo del otro ${ }^{43}$.

En esta crítica a la fenomenología husserliana radica toda la riqueza del argumento de Henry: la experiencia del otro no es susceptible de explicarse en base a la percepción o la intencionalidad de la conciencia, sino que encuentra su inteligibilidad en la afectividad. Henry señala:

39 Cf. Henry, Michel, Autodonation. Entretiens et conférences, Beauchesne, París, 2004, p. 164 .

40 Cf. Henry, Michel, Phénoménologie matérielle, op.cit., p. 139.

41 Henry, Michel, Phénoménologie matérielle, op.cit., p. 140.

42 Cf. Husserl, Edmund, Meditaciones cartesianas, op.cit., p. 153.

43 Cf. Henry, Michel, Phénoménologie matérielle, op.cit., p. 146. 
«La experiencia de la alteridad se falsea por entero en la medida en que el ego y el cuerpo que intervienen en dicha experiencia, en tanto cuanto la hacen posible, son un cuerpo y un ego constituidos. Pues la experiencia concreta y real de la alteridad es, como afirmaba el propio Husserl al comienzo de la $\mathrm{Me}$ ditación, un hecho trascendental que pertenece a mi esfera fenomenológica.» ${ }^{44}$

El fracaso de la percepción de la experiencia del otro en la fenomenología intencional radica en que la percepción es por naturaleza externa, es la percepción de un $\mathrm{Ob}$-jectum, en cambio, para Henry, se trata de una intersubjetividad viva y patética en la que estamos con el otro. La subjetividad es en primera persona, no se trata de la experiencia de una "cosa muerta», cuya cualidad psíquica no es más que una significación irreal asociada a su ser cósico. El despliegue y desarrollo de la intersubjetividad patética debe quedar regulado por las leyes del pathos de las subjetividades en su co-pertenencia al fondo de la vida, las leyes del sufrir y el gozar, del sentimiento, y no de la percepción como ocurre en la filosofía husserliana ${ }^{45}$.

La subjetividad, como afectividad, si bien genera dificultades, por ejemplo, respecto al problema del pensamiento y el lenguaje, nos abre, por otra parte, una interesante reflexión sobre la experiencia del otro, con las consecuencias éticas que ello conlleva, tal como vimos en el caso del cuerpo. La filosofía de Henry abre el horizonte y la posibilidad de una filosofía de la afectividad, ámbito tradicionalmente negado o excluido de la concepción de subjetividad. Comenzar a pensar la intersubjetividad como una experiencia esencialmente afectiva implica reflexionar y repensar sobre múltiples categorías del horizonte filosófico y sociológico, ya no desde el sentimiento como lo «inconsciente» o irracional, sino como una experiencia profundamente humana y por tanto subjetiva. Aun así, estos planteamientos nos siguen generando interrogantes respecto de cómo hacer una filosofía o teoría de la experiencia afectiva del otro, si ésta escapa al lenguaje y al pensamiento, que son las herramientas con que contamos para ello.

\section{El PROBLEMA DE LA COMUNIDAD EN LA FENOMENOLOGÍA DE LA VIDA}

El pensamiento de Henry suscita un debate relevante, así como dificultades y problemas no resueltos, que subyacen a su concepción de la vida social y la vida en común. En primer lugar, Henry reconoce que la vida social es producto del sujeto y, apoyándose en sus trabajos sobre Marx, denuncia la comprensión equivocada que hace el marxismo de la economía y la sociedad, que vuelve invisible al sujeto e hipostasia estructuras que sólo existen en la vida subjetiva. Las ciencias sociales, herederas en gran parte de la objetividad positivista y de la interpretación marxista de la historia, tienden a convertir en «cosa» lo social:

$44 \quad$ Ibid., p. 149.

45 Cf. Ibíd., p. 153. 
instituciones, estructuras, sistemas, y a darle con ello, una cierta autonomía e independencia de los sujetos que las constituyen. Henry nos recuerda:

«Son pues, las propiedades de esta subjetividad, las leyes de la vida, las que dan cuenta de las leyes de la economía, y es ante todo a partir de esta subjetividad viviente, que se construyen las realidades económicas que les sirven de equivalentes objetivos, sin que esta equivalencia y la racionalidad que la funda, puedan dejar de ser ilusorias.» ${ }^{46}$

Las determinaciones sociales pueden afectar al individuo sólo porque éstas son subjetivas, porque están fundadas esencialmente en la subjetividad. Esto significa que estas condiciones sociales son condiciones de la vida, no condiciones objetivas de una situación objetiva, abstracta y externa, sino condiciones que forman el tejido mismo y la sustancia de la vida, la sucesión de experiencias vividas y de sus vivencias ${ }^{47}$. El principal aporte de Henry, en este ámbito, es el reconocimiento del trabajo viviente como constituyente de la subjetividad, el que es entendido como el esfuerzo del propio cuerpo vivo y afectivo, que busca satisfacer las necesidades de la vida y que se constituye así en fundamento de la economía y la vida social.

En íntima relación y conexión con este problema, está el problema de la intersubjetividad y la posibilidad de la vida social, que Henry entiende desde el concepto de comunidad. Respecto de la intersubjetividad, como hemos visto, Henry propone que la experiencia del otro es posible y encuentra su inteligibilidad sólo en la afectividad. Para apoyar su posición presenta dos ejemplos de comunidades posibles, ambos bastante problemáticos, pues aquello que tienen en común no tiene nada que ver con la percepción ${ }^{48}$. El primer ejemplo nos remite al arte y se expresa en la comunidad conformada por los admiradores de Kandinsky, quienes aún sin conocerse, y por tanto sin ser objeto de percepción unos de otros, comparten la fuerza invisible del pathos de la obra creada por el artista convirtiéndose así en comunidad ${ }^{49}$. El otro ejemplo es más complejo aún y refiere a la "comunidad con los muertos», pues conservamos la huella del recuerdo y compartimos ese pathos que se sustrae a nuestros actos de pensamiento, aun cuando el soporte perceptivo ha sido abolido ${ }^{50}$. Esta comunidad con los muertos, si bien en el mundo moderno no es considerada como forma de comunidad, sí tuvo un papel importante en épocas pasadas y podría tenerla, según Henry, en sociedades tradicionales hoy en día ${ }^{51}$. Ambos ejemplos se presentan, sin duda, problemáticos y confusos a la hora de pensar la intersubjetividad, la comunidad y la vida social en tanto comunidad.

\footnotetext{
46 Henry, Michel, Phénoménologie de la vie, Tome I De la phénoménologie, op. cit., p. 57.

47 Cf. Henry, Michel, Marx, Gallimard, París, 1976, pp. 242-243.

48 Cf. Fainstein, Graciela, op.cit., p. 223.

49 Cf. Henry, Michel, Phénoménologie matérielle, op.cit., pp. 153-154.

50 Cf. Ibid., pp. 154-155.

51 Cf. Ibid.
} 
Si bien Henry intenta hacer un aporte original a partir de su crítica radical a la concepción husserliana de la intersubjetividad, ésta aparece en parte como una crítica un tanto exagerada e injusta, pues en ningún caso pareciera que Husserl haya dejado cerrados estos problemas y posiciones contra las que Henry acomete tan duramente ${ }^{52}$. Éste es precisamente uno de los elementos que ha levantado polémica acerca de las interpretaciones que Henry hace de Husserl.

En el segundo apartado de su capítulo "Pathos-con», contenido en Phénoménologie Materielle, Henry aborda la idea de comunidad intentando ir más allá de los análisis husserlianos de la Quinta meditación, donde se habría dado por supuesta la comunidad originaria, por parte de Husserl, cuando un ser está con otro ${ }^{53}$. Henry deja la última palabra respecto de la posibilidad de comunidad a un misterio, el que quedará vinculado finalmente a la vida. El hecho de que el problema de la intersubjetividad se aborde en términos de comunidad, se debe fundamentalmente a que Henry se plantea como tarea continuar el camino en el punto donde Husserl lo ha dejado, es decir, en el descubrimiento que permite considerar la existencia de una esfera originaria donde las «mónadas» que son los «ego» (el propio y el del otro) podrían abrirse y comunicarse ${ }^{54}$.

Para Henry la constatación de la realidad que comparten los miembros de una comunidad, su esencia puesta en común, es la vida. La esencia de toda comunidad es la vida y por tanto toda comunidad es "comunidad de vivientes». No se trata simplemente de «una cosa en común», sino a la inversa, la vida es el modo de revelación, por eso es la esencia de toda manifestación:

«La vida es la auto-donación en un sentido radical, en el sentido de que ella es lo dado en dicho don, sólo en ella tenemos acceso a ella. Ningún camino conduce a la vida sino la vida misma. En la vida ningún camino conduce fuera de ella, es decir, no permite a lo vivo dejar de serlo. La vida es la subjetividad absoluta en tanto cuanto se experimenta a sí misma y no es otra cosa, el puro hecho de experimentarse a sí misma inmediatamente y sin distancia. He aquí pues lo que constituye la esencia de toda comunidad posible, lo que está en común.» ${ }^{55}$

Lo que tienen en común los miembros de la comunidad de vivientes es, precisamente, la vida, y éstos no son algo extrínseco a ella. De este modo se entrelazan en el pensamiento de Henry los conceptos de vida, individuo viviente y comunidad, y vemos, bajo este análisis, el sinsentido de oponer individuo y comunidad, como lo han hecho diversos sistemas políticos y teorías. Para Henry en cambio:

«La comunidad no es otra cosa que este conjunto de individuos vivos. El concepto de individuo, en el sentido aquí destacado, es tan esencial que sólo hay comunidad con él. La tentativa de oponer el uno a la alteridad, la

52 Cf. FaInStein, Graciela, op.cit., p. 227.

53 Cf. Husserl, Edmund, Meditaciones cartesianas, op.cit., pp. 152-153.

54 Cf. Fainstein, Graciela, op.cit., p. 228.

55 Henry, Michel, Phénoménologie matérielle, op.cit., pp. 161-162. 
comunidad y el individuo, de establecer entre ellos una relación de jerarquía, es un simple sinsentido: dicha tentativa opone la esencia de la vida, lo implicado necesariamente por ella. ${ }^{56}$

La oposición individuo-comunidad solo puede darse en la objetividad del mundo, que ve estos conceptos como representación, es decir, en el objetivismo teórico o político. Si aquello que se tiene en común es la vida como esencia de la comunidad, y ésta no se muestra en la representación de la objetividad, nos preguntaremos, junto con Henry, si toda comunidad es por principio «invisible». Para responder a esta pregunta Henry vuelve sobre el análisis y la crítica de la fenomenología de la percepción y la intencionalidad. Para la fenomenología histórica (la fenomenología husserliana), la comunidad sería comunidad de los que perciben uno y el mismo mundo objetivo, y por tanto, lo que tendrían en común, sería la objetividad del mundo y no la vida de la que participan.

Que la fenomenología tradicional haya recurrido al concepto de apercepción asemejante o analógica ${ }^{57}$ para explicar el acceso de mi ego al ego del otro, descartando la percepción, es para Henry la prueba de la dificultad que supone el hecho de que su ipseidad o subjetividad absoluta no pueda ser percibida, pues no es algo que está en el afuera, en el mundo ${ }^{58}$. Incluso frente a los análisis de Scheler, Henry connota el fracaso de la fenomenología intencional, insistiendo en que la relación de vivientes sólo puede comprenderse a partir de la esencia de la vida. La vida dará la clave comprensiva de cualquier relación entre vivientes ${ }^{59}$.

Nuevamente en este texto Henry nos ofrece ejemplos de comunidad que resultan problemáticos y discutibles desde el punto de vista de las mismas experiencias que refieren, pero que estarían fuera de la relación objetiva o intencional: la relación madre-hijo ${ }^{60}$, la relación hipnotizador-hipnotizado, la relación entre psicoanalista y psicoanalizado fundamentalmente respecto a la transferencia. Lo que quiere connotar Henry con estos ejemplos, por cierto discutibles, es la afectividad, la fuerza de la experiencia que se experimenta a sí misma en la vida, lo único que subsiste como verdadero cuando ponemos entre paréntesis el mundo:

«Toda comunidad es por esencia afectiva y, al mismo tiempo, pulsional —ello atañe no sólo a las comunidades fundamentales de la sociedad, la pareja, la familia, sino a toda comunidad en general, cualesquiera que sean sus intereses y sus motivaciones explícitas. ${ }^{61}$

Ibid., p. 163.

Cf. Husserl, Edmund, Meditaciones cartesianas, op.cit., pp. 171-185.

Cf. Henry, Michel, Phénoménologie matérielle, op.cit., p. 170.

Cf. Ibid., p. 222.

Cf. Ibíd.

Henry, Michel, Phénoménologie matérielle, op.cit., p. 175. 
En la vida, y por su auto-afección, nace cada vez la experiencia de sí que es un viviente. La salida al problema de la comunidad nos lleva prácticamente a una dimensión mística:

«Tal es el misterio de la vida: que el viviente es co-extensivo al todo de la vida en él, que todo en él es su propia vida. El viviente no se funda él mismo, tiene un Fondo que es la vida, pero este Fondo no es diferente de él, es la auto-afección en la que se auto-afecta y con la que esta forma se identifica. ${ }^{62}$

En síntesis, cada uno de los miembros de la comunidad se relaciona con el otro en la Vida, antes de que lo haga en el mundo, pues la Vida es el fondo común donde ambos participan de modo que están «abismados en lo Mismo» ${ }^{63}$. Henry define finalmente la comunidad como «napa afectiva subterránea» ${ }^{64}$, de modo que cada uno bebe de esa fuente y en ese pozo que es él mismo, sin saberlo, sin distinguirse de sí mismo, del otro y del Fondo.

Henry nos conduce a un camino complejo y, aunque podemos valorar desde una perspectiva ética el carácter apriorístico y común del ser de todos los vivientes, esto no nos dice nada respecto de cómo esa napa subterránea que es la vida, se expresa en la vida cotidiana y nos permite vivir con otros, relacionarnos en el mundo de la vida compartido, no sólo en tanto iguales, sino también en tanto diferentes. La aporía se presenta, en la filosofía de Henry, al intentar pensar la comunidad sin apelar al misterio o a la mística, lo que podría estar vinculado al curso posterior de su pensamiento dedicado a la filosofía del cristianismo.

Es evidente que el camino abierto por Henry es una aproximación creativa, interesante y provocadora para afrontar problemas recurrentes en la filosofía y las ciencias sociales, por lo que se transforma en una motivación para seguir profundizando en la tarea fenomenológica: aprender a ver aquello que la vida ya está mirando ${ }^{65}$ y más aún, ya está viviendo en su pathos subjetivo. Sin duda el diálogo entre fenomenología y ciencias sociales también tiene mucho camino por recorrer y requerirá de diversos aportes, trabajos y miradas del problema, sobre todo si coincidimos con Henry en que una filosofía de la subjetividad, y por ende de la intersubjetividad, es una tarea que está aún por hacerse.

$62 \quad$ Ibid., p. 177.

63 Ibíd., p. 178.

64 Cf. Ibíd., p. 178.

65 Cf. García-Baró, Miguel, Vida y mundo. La práctica de la fenomenología, Trotta, Madrid, 1999, p. 14. 


\section{BiBLIOGRAFÍA}

Dufour-Kowalska, G. (2003). Michel Henry: passion et magnificence de la vie. París: Beauchesne.

Fainstein, G. (2009). Michel Henry: una fenomenología de la afectividad o una filosofía en el limite del pensamiento, Tesis para la obtención del grado de Doctor, Pontificia Universidad Comillas de Madrid, Madrid.

García-Baró, Mi. (1999). Vida y mundo. La práctica de la fenomenología. Madrid: Trotta. Henry, M. (1963). L'essence de la manifestation. París: PUF.

- (1965). Philosophie et phénoménologie du corps. París: PUF.

- (1976). Marx, Gallimard, París.

- (1987). La Barbarie. París: PUF.

- (1990)., Phénoménologie matérielle. París: PUF.

- (2000). Incarnation. París: Seuil.

- (2003). Phénoménologie de la vie, Tome I De la phénoménologie. París: PUF.

- (2003). Phénoménologie de la vie, Tome II De la subjectivité. París: PUF.

- (2004). Phénoménologie de la vie, Tome III De l'art et du politique. París: PUF.

- (2004). Phénoménologie de la vie, Tome IV Sur l'éthique et la religion. París: PUF.

- (2004). Autodonation. Entretiens et conférences. París: Beauchesne.

- (2005). Voir l'invisible. Sur Kandinsky. París: PUF.

- (2010). Fenomenología de la Vida. Buenos Aires: Prometeo.

Husserl, E. (1985). Meditaciones cartesianas. Madrid: Fondo de Cultura Económica.

Leclercq, J. y Henry, A. (2009). «Biographie en forme d'entretien», en Cahier Michel Henry, Paris-Lausanne : Lâge de l'homme.

Leclercq, J. (2016). «Afectividad, pasividad y experiencia pura. Reflexiones sobre el proyecto de Michel Henry», en Lipsitz, M. y Belvedere, C., Problemas de fenomenología material. Buenos Aires: UNGS.

Lipsitz, M. y Belvedere, C. (2016). Problemas de fenomenología material. Buenos Aires: UNGS.

Moser, V. (2011). «Avec Michel Henry: pour une monadologie radicale», en Les cahiers philosophiques de Strasbourg, $\mathrm{n}^{\circ} 30$.

Pontificia Universidad Católica de Valparaíso

Myriam Díaz ERbetta myriam.diaz.e@pucv.cl

[Artículo aprobado para publicación en enero de 2018] 
